\title{
Two-year patient-related outcome measures (PROM) of primary ventral and incisional hernia repair using a novel three-dimensional composite polyester monofilament mesh: the SymCHro registry study
}

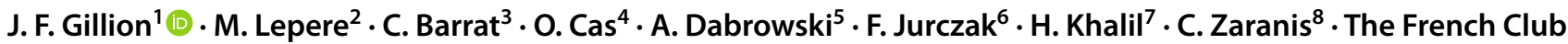 \\ Hernie Study Group
}

Received: 2 October 2018 / Accepted: 2 March 2019 / Published online: 18 March 2019

(c) The Author(s) 2019

\begin{abstract}
Purpose This study examined patient-related outcome measures (PROMs) after repair of ventral primary or incisional hernias using Symbotex ${ }^{\mathrm{TM}}$ composite mesh (SCM), a novel three-dimensional collagen-coated monofilament polyester textile. Methods Pre-operative, peri-operative, and post-operative data were obtained from the French "Club Hernie" registry with 12- and 24-month follow-up.

Results One-hundred consecutive patients (mean age $62.0 \pm 13.7 ; 51 \%$ female) underwent repair of 105 hernias: primary $\left(39 / 105,37.1 \%\right.$, defect area $\left.5.2 \pm 5.6 \mathrm{~cm}^{2}\right)$ and incisional $\left(66 / 105,62.9 \%, 31.9 \pm 38.7 .8 \mathrm{~cm}^{2}\right)$. The mean BMI was 29.7 $\left( \pm 5.6 \mathrm{~kg} / \mathrm{m}^{2}\right)$. American Society of Anesthesiologists classifications were I 39.4\%, II 37.4\% and III 23.2\%.75\% had risk factors for healing and/or dissection. Of 38 primary repairs, 37 were completed laparoscopically (combined approach $n=1$ ), and of 62 incisional hernia repairs, 40 were completed laparoscopically, and 20 by open repair (combined approach $n=2$ ). Laparoscopic was quicker than open repair $(36.2 \pm 23.5 \mathrm{~min}$ vs. $67.4 \pm 25.8, p<0001)$. Before surgery, $86.3 \%$ of hernias were reported to cause discomfort/pain or dysesthesia. At 24 months (93 of 100 patients), 91 (97.8\%) reported no lump and $81(87.1 \%)$ no pain or discomfort. Of 91 patients, $86(94.5 \%)$ rated their repair "good" or "excellent." There were nine nonserious, surgeon-detected adverse events (ileus, $n=3$; seroma, $n=6$ ) and one hernia recurrence (6-12 months).

Conclusions Compared to baseline, open and laparoscopic surgery improved PROMs 24 months after primary and incisional hernia repair. Minimal complications and recurrence support the long-term efficacy of SCM.
\end{abstract}

Keywords Hernia repair $\cdot$ Ventral hernia $\cdot$ Incisional hernia $\cdot$ Abdominal hernia $\cdot$ Laparoscopy $\cdot$ Surgical mesh

\section{Barrat: Deceased.}

The members of "The French Club Hernie Study Group" are listed in acknowledgement section.

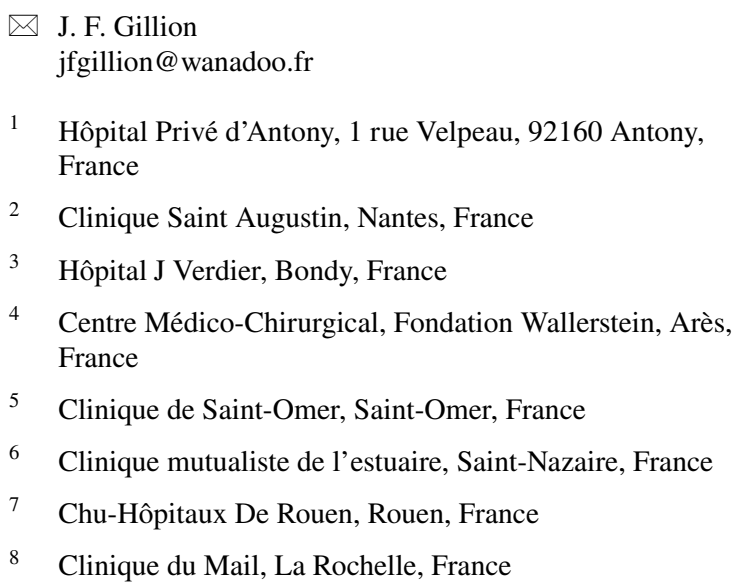

\section{Introduction}

Primary ventral and incisional hernias of the abdominal wall are common, pose significant medical issues, and are associated with an economic burden [1,2]. They may be unsightly, cause pain, interfere with professional activities and have an impact on quality of life (QoL). While up to one-third of hernias is asymptomatic, surgical repair is generally recommended to reduce the risk of obstruction and strangulation [3].

The laparoscopic approach to primary ventral and incisional hernia repair is increasingly popular, offering the potential advantages of similar recurrence rates but fewer post-operative complications in selected patients [4]. A Cochrane Collaboration review of ten randomized controlled trials demonstrated that laparoscopic surgery was associated with fewer wound infections, improved cosmesis, and a 
shorter hospital stay [5]. Outcomes are also widely accepted as being improved by the incorporation of mesh in both open and laparoscopic procedures [6]. Incorporating mesh appears to offer advantages even for small defects such as primary umbilical hernias $\leq 4 \mathrm{~cm}$ in width [7].

Recurrence after surgery is influenced by several patient factors, including obesity, fitness, and defect size [3, 8]. Similarly, technical factors and the choice of mesh can affect outcomes after primary ventral or incisional repair. For example, recurrence rates are reported to be lower where there is mesh overlap of at least $5 \mathrm{~cm}$ and there is a high mesh area-to-defect area $(M / D)$ ratio $[9,10]$.

Although the trend has moved away from mesh placement inside the abdominal cavity due to long-term complications related to adhesion formation, most laparoscopic ventral hernia repairs worldwide still employ an intraperitoneal onlay mesh (IPOM) technique. When using an intraperitoneal approach, reducing adhesion formation and prevention of damage to adjacent viscera are key aims [11]. Device manufacturers have sought to minimize this problem by including a continuous protective layer in their mesh designs. One early product was Parietex composite mesh (PCO, Covidien LP, Trevoux, France, a wholly owned subsidiary of Medtronic plc). First introduced in 1998, this multifilament polyester associated with a strong safety profile and minimal adhesion formation proved a popular repair option with over a million units sold to date [12]. Suggestions from surgeons regarding desired handling characteristics and memory shape resulted in a change from a multi- to a monofilament polyester-knitted structure and the development of Symbotex ${ }^{\mathrm{TM}}$ composite mesh (SCM; Covidien LP).

SCM is a novel three-dimensional monofilament polyethylene terephthalate (polyester) textile. On its visceral surface is a modified absorbable hydrophilic film consisting of a mixture of collagen and glycerol [13]. Other claimed design features include increased conformability, transparency, green centering marking, and a green flap, all designed to help visualize the fixation area and facilitate accurate position of the prosthesis against the abdominal wall.

Results, 12 months after laparoscopic or open mesh repair, suggest that the use of SCM is safe and effective [14]. The present study, an update of this registry study, aimed to examine outcomes 24 months after surgery with reference to factors which are important to patients, e.g., have their symptoms resolved compared to baseline and are they satisfied with the outcome of their treatment. The need to focus on patient-reported outcome measures (PROMs) is increasingly recognized [15]. From a surgical perspective, the researchers also sought to examine the effect of the $M / D$ ratio on hernia recurrence [10]. Because primary ventral and incisional hernias have been shown to differ in terms of patient characteristics and post-operative complications, data are presented and analyzed by subgroup [16].

\section{Methodology}

\section{Overview}

This prospective study was performed by the Club Hernie, a group of approximately 40 French surgeons specialized in parietal surgery. An online de-identified, encrypted registry was established by the group in 2011 to prospectively record all consecutive inguinal and ventral hernia operations in real time, ahead of any outcomes being known. The registry is comprised of 164 input boxes with predefined questions and answers that were chosen by a medical advisory board before collection began. All answers must be selected to enable patient entry onto the registry which promoted a high completion rate. Standard, anonymized data capture includes pre-operative, peri-operative, and post-operative details.

All Club Hernie members are required to sign a quality charter promising to provide detailed information and full disclosure.

A proportion of participating surgeons employed Symbotex ${ }^{\mathrm{TM}}$ composite mesh (SCM) in primary ventral and incisional hernia repair. The present analysis focused on the first 100 adult subjects included in the registry who underwent laparoscopic or open ventral hernia repair using SCM between July 2014 and May 2015.

This investigation was registered on http://www.clinicaltr ials.gov (NCT02206828). The French national ethics committee, the Comité de Protection des Personnes (CPP), was informed, and it subsequently issued a study waiver (CPP Sud Est III Lyon-File QH 15/2014) due to the registry nature of the study. Covidien LP provided technical support to the registry on a pro bono basis but did neither influence contents, patient results nor data interpretation.

\section{Study aims, objectives and endpoints}

The aim of this observational registry study was to assess the short- and long-term clinical outcomes following the use of SCM in primary and incisional abdominal wall hernia surgeries by open or laparoscopic approach according to instructions for use.

The primary objectives (and endpoints) were to evaluate the incidence of peri-operative and post-operative complications, hernia recurrence and PROM during the first 2 years of follow-up. Secondary objectives (endpoints) included assessment of surgical techniques, mesh fixation and handling, operative time, surgeon satisfaction and length of stay. Issues of importance to patients included QoL, individual satisfaction and pain assessment. 


\section{Patients and sample size}

Consecutive patients $\geq 18$ years of age undergoing elective or emergency primary ventral and/or incisional hernia repair were included irrespective of the size and complexity of their defects. There were no exclusion criteria. The chosen sample size of 100 was based on previous experience using PCO mesh, which suggested that a recurrence rate below $6 \%$ could be expected after open or laparoscopic ventral hernia repair, with a standard $95 \%$ confidence interval and an estimated $15 \%$ loss to follow-up [17, 18]. Fitness for operation was assessed based on American Society of Anesthesiologists (ASA) physical status scores [19].

During a pre-operative visit, the responsible surgeon gave all patients a written notice informing them about the nature and purpose of the study. Because of the CPP waiver, completion of an informed consent form was not required.

\section{Operative treatment}

Whether patients underwent laparoscopic or open repair of their primary ventral or incisional hernia using SCM was at the discretion of the surgeon. Similarly, the type of fixation was not imposed but recorded. The mesh was trimmed if necessary while ensuring sufficient overlap to cover the adjacent edges of the defect. An overlap greater than $5 \mathrm{~cm}$ has previously been shown to reduce the rate of recurrence [9]. Skin-to-skin operating time and time required to fix the mesh were recorded, along with surgeons' views on various aspects of the procedure.

\section{Hernia defect and mesh size}

Dimensions of the hernia defects $\left(\mathrm{cm}^{2}\right)$ were calculated using a circular area formula for primary hernias and an elliptical formula for incisional hernias [20]. A similar formula was used for mesh size. Based on these dimensions, $M / D$ ratios were calculated.

\section{Choice of Symbotex composite mesh}

Surgeons were permitted to choose an appropriate size and shape from the three available types of SCM: a flat sheet without sutures (SYM), a flat sheet with prepositioned sutures (SYM-F), and a flat sheet with an open textile flap (SYM-OS) designed to aid placement and fixation.

\section{Follow-up}

Patients were followed up on at set points after surgery (Fig. 1). Pain assessment was performed by a nurse on the day of surgery, on Day 1, and on Day 8, either directly or

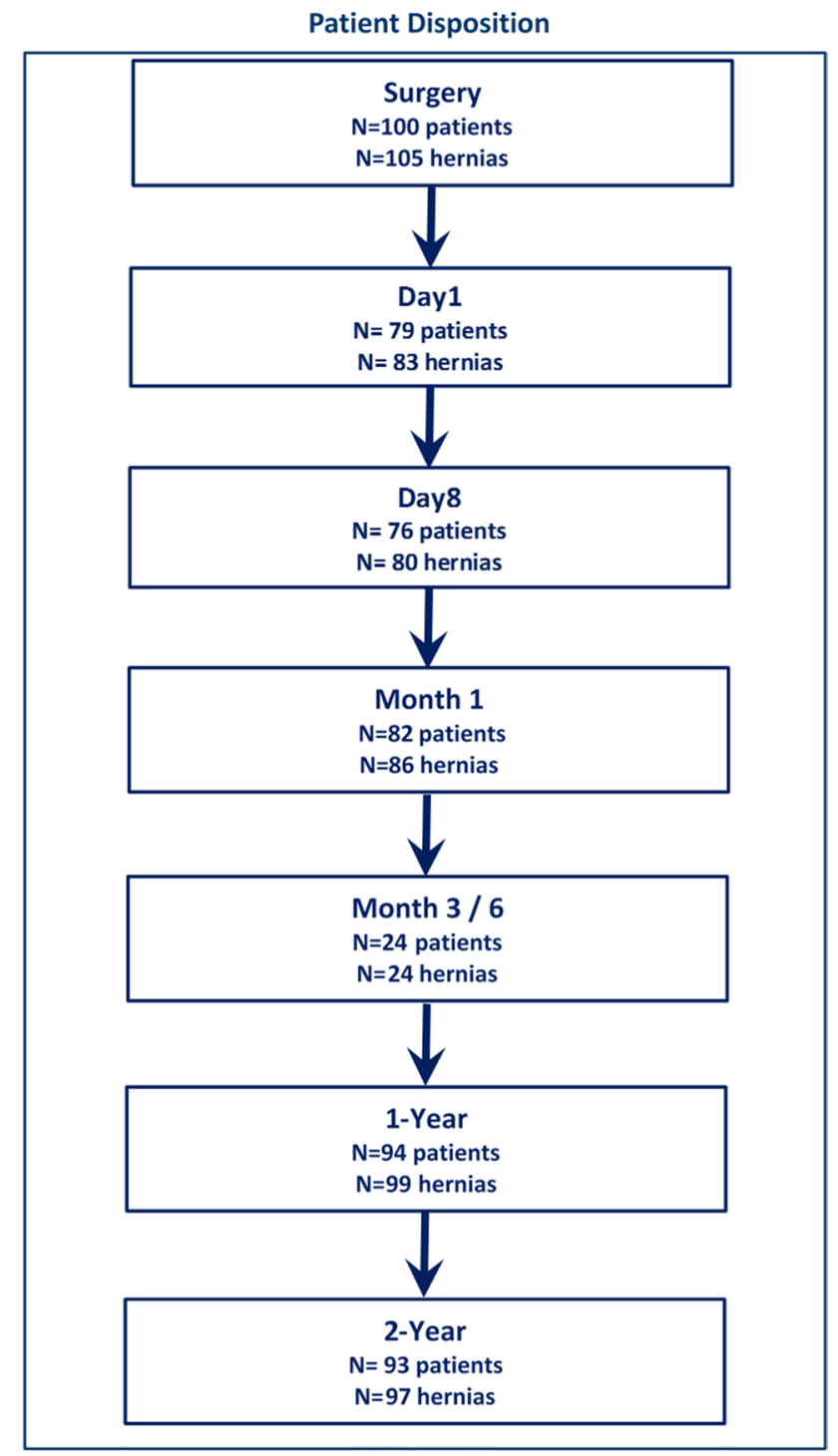

Fig. 1 Study flow chart. Schedule of patient follow-up and number involved at each time point

by telephone if the patient had been discharged. Additional pain assessment was performed by the surgeon during the patient's 1-month review appointment. All patients experiencing symptoms (e.g., pain or a bulge) were advised to attend a further follow-up appointment at 3 months. Follow-up at the end of year 1 and year 2 involved a detailed QoL questionnaire administered via telephone by a Club Hernie clinical research assistant acting independently of the operating surgeon [21]. The information was entered verbatim in the registry, without interpretation or adjustment.

Those patients from whom a response could not be elicited after five attempts were considered lost to follow-up to minimize non-response bias. 


\section{Data presentation and analysis}

Descriptive data per patient, hernia, and surgeon are presented numerically, in terms of means, standard deviations, and ranges. Missing values are mentioned in the relevant tables. Categorical variables are presented as frequencies and percentages of the number of recorded entries. A minority of patients had more than one hernia; where both types of hernia existed, their classification as primary or incisional was at the surgeon's discretion.

Statistical analysis was performed using Minitab v15.0 and SAS 9.2. Comparative group analysis was performed using Student's $t$ test and Wilcoxon's rank-sum test for nonparametric data. Qualitative data were examined using Pearson's chi-squared test and Fisher's exact test, as appropriate.

Pain was assessed at intervals during the study using a visual analog scale (VAS). Comparison of paired data was performed using Student's $t$ test and Wilcoxon's signed-rank test.

\section{Results}

\section{Patient demographics}

One-hundred consecutive patients (mean age 62.0 \pm 13.7 ; $51 \%$ female) who entered into the Club Hernie registry between July 2014 and May 2015 underwent repair of 105 hernias. 39/105 were primary $(37.1 \%$, defect area $5.2 \pm 5.6 \mathrm{~cm}^{2}$ ) and the remaining $66 / 105$ were incisional $\left(62.9 \%, 31.9 \pm 38.7 \mathrm{~cm}^{2}\right)$. The mean BMI was $29.7( \pm 5.6 \mathrm{~kg} /$ $\mathrm{m}^{2}$ ). ASA classifications were I $39.4 \%$, II $37.4 \%$ and III $23.2 \% .75 \%$ of patients had risk factors for healing and/or dissection. Patients with an incisional hernia were older (mean age $63.4 \pm 13.1$ years vs. $58.1 \pm 13.8$ years; $p=0.026$ ) and tended to report a hernia history $(32 / 62,51.6 \%$ vs. $4 / 38$, $10.5 \%$; $p<0.0001$; Table 1).

In patients classified as having an incisional hernia, these defects were predominantly situated in the midline $(n=47$, epigastric $n=15$, peri-umbilical $n=14$, sub-umbilical $n=6$, supra-pubic $n=1$, mixed $n=12$ ) or laterally ( $n=12$, sub-costal $n=3$, flank $n=3$, iliac $n=4$, lumbar $n=2$ ). Three patients were reported as having both midline and lateral hernias. By comparison, in patients with a primary hernia, 30/38 were classified as having an umbilical/sub-umbilical defect. Other types were epigastric $(n=6)$, Spigelian $(n=1)$, and mixed (umbilical/sub-umbilical and epigastric $n=1$ ).

\section{Pre-operative symptoms}

Information on pre-operative symptoms was available for 102 of 105 hernias (missing values $n=3$; Table 2). While 7 of 102 hernias (6.9\%; primary $n=2$; incisional $n=5$ ) were asymptomatic, most operated hernias caused discomfort, pain, or dysesthesia $(88 / 102,86.3 \%)$. Seven hernias (6.9\%) were described as strangulated. In five, the contents of the hernias were reducible by taxis, and in one, the hernia was strangulated without bowel occlusion. In the remaining patient, there was evidence of strangulation and occlusion.

\section{Hernia details}

A total of 105 hernias were treated. 39 of $105(37.1 \%$,) hernias were classified as primary, and 66 of $105(62.9 \%)$ as incisional. Four patients had more than one separate hernia: two patients had two incisional hernias, one patient had both a primary and incisional hernia (classified as incisional), and one patient had three incisional hernias.

At operation, defects appeared to be multifocal in 21 of 104 hernias (20.2\%; unifocal $n=83,79.8 \%$; missing value $n=1$; Table 2). Thirty-two of 103 hernias were reported as incarcerated (31.1\%; not incarcerated $n=71,68.9 \%$; missing values $n=2)$.

Primary hernia defects $(n=39)$ were found to be smaller than incisional hernia defects $(n=65$, missing value $n=1)$ in width $(2.3 \pm 1.0 \mathrm{~cm}$ vs. $4.9 \pm 2.6 \mathrm{~cm} ; p<0.0001)$, length $(2.5 \pm 1.2 \mathrm{~cm}$ vs. $6.5 \pm 4.5 \mathrm{~cm} ; p<0.0001)$, and total defect area $\left(5.2 \pm 5.6 \mathrm{~cm}^{2}\right.$ vs. $31.9 \pm 38.7 \mathrm{~cm}^{2} ; p<0.0001$; Table 2).

\section{Surgical approach}

Surgical repair using SCM was performed at 14 French centers. All procedures were under general anesthetic. Three operations were classified as an emergency (primary $n=1$; incisional $n=2$ ). All but three operations were considered "clean" ("clean contaminated": primary $n=1$; incisional $n=2$ ). In 37 of 38 patients with a primary hernia, repair was completed laparoscopically, with one case requiring a combined open and laparoscopic approach (Table 3). In 40 of 62 patients (64.5\%) with an incisional hernia, the operation was planned and completed laparoscopically. Similarly, in 20 of 62 (32.3\%) patients, the operation was planned and completed as an open procedure. 2 of 62 incisional hernia repairs involved a hybrid open and laparoscopic approach.

Larger incisional hernias tended to be operated on via an open approach (laparoscopic repair mean defect size $17.3 \mathrm{~cm}^{2} \pm 20.2$ vs. open repair $59.5 \mathrm{~cm}^{2} \pm 50.5 ; p<0.0001$ ), took longer to perform, and involved patients spending more time in the hospital (see below).

\section{Operative duration, time for mesh placement, and hospital stay}

Overall, laparoscopic repair was quicker than open repair $(36.2 \pm 23.5 \mathrm{~min}$ vs. $67.4 \pm 25.8, p<0.0001$; Table 4$)$ and laparoscopy patients went home earlier $(1.6 \pm 1.6$ hospital 
Table 1 Patient demographics, including body mass index (BMI), smoking status, American Society of Anesthesiologists (ASA) physical status score, hernia history, risk factors related to surgical dissection and healing $(n=100)$

\begin{tabular}{|c|c|c|c|c|}
\hline Criteria & $\begin{array}{l}\text { Overall patients } \\
(n=100)\end{array}$ & $\begin{array}{l}\text { Primary hernia } \\
\text { patients }(n=38)\end{array}$ & $\begin{array}{l}\text { Incisional hernia } \\
\text { patients }(n=62)\end{array}$ & $p$ value \\
\hline \multicolumn{5}{|l|}{ Gender } \\
\hline Male & $49(49.0)$ & $22(57.9)$ & $27(43.5)$ & \multirow[t]{2}{*}{0.1640} \\
\hline Female & $51(51.0)$ & $16(42.1)$ & $35(56.5)$ & \\
\hline Age (years) & $62(13.7)$ & $58.1(13.8)$ & $63.4(13.1)$ & 0.0260 \\
\hline BMI $\left(\mathrm{kg} / \mathrm{m}^{2}\right)$ & $29.7(5.6)$ & $30.4(6.1)$ & $29.3(5.2)$ & 0.377 \\
\hline Missing data & 1 & 1 & 0 & \\
\hline \multicolumn{5}{|l|}{ Smoking Status } \\
\hline Non-smoker (never smoked or stopped $\geq 12$ months) & $83(84.7)$ & $33(86.8)$ & $50(83.3)$ & \multirow[t]{3}{*}{0.631} \\
\hline Smoker (current, recent, regular or occasional smoker) & $15(15.3)$ & $5(13.2)$ & $10(16.7)$ & \\
\hline Missing data & 2 & 0 & 2 & \\
\hline \multicolumn{5}{|l|}{ ASA classification } \\
\hline Class I & $39(39.4)$ & $24(64.9)$ & $15(24.2)$ & \multirow[t]{5}{*}{0.0003} \\
\hline Class II & $37(37.4)$ & $8(21.6)$ & $29(46.8)$ & \\
\hline Class III & $23(23.2)$ & $5(13.5)$ & $18(29.0)$ & \\
\hline Class IV/V & $0(0.0)$ & $0(0.0)$ & $0(0.0)$ & \\
\hline Missing data & 1 & 1 & 0 & \\
\hline \multicolumn{5}{|l|}{ Hernia history } \\
\hline None & $64(64.0)$ & $34(89.5)$ & $30(48.4)$ & \multirow[t]{2}{*}{$<0.0001$} \\
\hline Previous history (one or more) & $36(36.0)$ & $4(10.5)$ & $32(51.6)$ & \\
\hline \multicolumn{5}{|l|}{ Risk factors related to surgical dissection } \\
\hline No & $31(31.3)$ & $21(56.8)$ & $10(16.1)$ & \multirow[t]{3}{*}{$<0.0001$} \\
\hline Yes (one or more) & $68(68.7)$ & $16(43.2)$ & $52(83.9)$ & \\
\hline Missing data & 1 & 1 & 0 & \\
\hline \multicolumn{5}{|l|}{ Risk factors related to healing } \\
\hline No & $65(66.3)$ & $30(78.9)$ & $35(58.3)$ & \multirow[t]{8}{*}{0.0354} \\
\hline Yes (one or more) & $33(33.7)$ & $8(21.1)$ & $25(41.7)$ & \\
\hline $\begin{array}{l}\text { Anticoagulant treatment or spontaneous coagulation/bleed- } \\
\text { ing disorder }\end{array}$ & $18(18.4)$ & $4(10.5)$ & $14(23.7)$ & \\
\hline Chemotherapy/immunosuppressive treatment & $8(8.2)$ & $1(2.6)$ & $7(11.9)$ & \\
\hline Diabetes & $7(7.1)$ & $0(0.0)$ & $7(11.9)$ & \\
\hline Corticosteroids & $4(4.1)$ & $0(0.0)$ & $4(6.8)$ & \\
\hline Other (unspecified) & $9(9.2)$ & $3(7.9)$ & $6(10.0)$ & \\
\hline Missing data & 2 & 0 & 2 & \\
\hline \multicolumn{5}{|l|}{ Risk factors related to healing and/or surgical dissection } \\
\hline No & $25(25.0)$ & $18(47.4)$ & $7(11.3)$ & \multirow[t]{2}{*}{$<0.0001$} \\
\hline Yes (one or more) & $75(75.0)$ & $20(52.6)$ & $55(88.7)$ & \\
\hline
\end{tabular}

Results are expressed in $\mathrm{n}(\%)$ for categorical data and mean (SD) for quantitative data; $p$ value $<0.05$ is significant

$B M I$ Body Mass Index, ASA American Society of Anesthesiologists physical status score

days vs. $4.7 \pm 2.0, p<0.0001)$. Similarly, repairing an incisional hernia laparoscopically took less time than an open repair (41.6 \pm 24.0 min vs. $67.4 \pm 25.8 ; p<0.0001$; Table 4$)$ and was associated with a shorter inpatient stay $(2.2 \pm 1.7$ hospital days vs. $4.7 \pm 2.0, p<0.0001)$. A total of 20 procedures were ambulatory (primary $15 / 36$ vs. incisional $5 / 56$; $p<0.0001$; missing $n=8$ ).

All meshes were positioned intraperitoneally including one partially inserted in the preperitoneal space. The mean time required to position the mesh was $9.4 \pm 6.3 \mathrm{~min}$ (laparoscopic $8.6 \pm 5.5$ vs. open $10.1 \pm 5.8$; Table 2). 83 (39 primary and 44 incisional) of the 105 hernias were repaired laparoscopically. Fascial closure was achieved in 26 of these 83 cases (31\%), in 9 of 39 primary (23\%), and 17 of 44 incisional $(39 \%)$ hernias $\left(\chi^{2}=2,33\right)$. Fascial closure was also more frequent in larger defects $\left(15.3 \mathrm{~cm}^{2} \pm 10.3\right.$ vs. $4.7 \mathrm{~cm}^{2} \pm 2.8 ; p=0.0011$ ). The mesh fixation consisted of stapling using absorbable (61 cases) and non-absorbable 
Table 2 Hernia details, including symptoms and dimensions $(n=105)$

\begin{tabular}{|c|c|c|c|c|}
\hline Criteria & $\begin{array}{l}\text { Overall her- } \\
\text { nias }(n=105)\end{array}$ & $\begin{array}{l}\text { Primary her- } \\
\text { nias }(n=39)\end{array}$ & $\begin{array}{l}\text { Incisional Her- } \\
\text { nias }(n=66)\end{array}$ & $p$ value \\
\hline \multicolumn{5}{|l|}{ Multisite hernia } \\
\hline No & $83(79.8)$ & 34 (89.5) & $49(74.2)$ & \multirow[t]{3}{*}{0.0624} \\
\hline Yes & $21(20.2)$ & $4(10.5)$ & $17(25.8)$ & \\
\hline Missing data & 1 & 1 & 0 & \\
\hline \multicolumn{5}{|l|}{ Hernia symptoms } \\
\hline Asymptomatic hernia & $7(6.9)$ & $2(5.1)$ & $5(7.9)$ & \\
\hline Discomfort/pain or pre-operative dysesthesia & $88(86.3)$ & $34(87.2)$ & $54(85.7)$ & \\
\hline Hernia reducible by taxis & $5(4.9)$ & $2(5.1)$ & $3(4.8)$ & \\
\hline Strangulated hernia without occlusion & $1(1.0)$ & $1(2.6)$ & $0(0.0)$ & \\
\hline Strangulated hernia with occlusion & $1(1.0)$ & $0(0.0)$ & $1(1.6)$ & \\
\hline Missing data & 3 & 0 & 3 & \\
\hline \multicolumn{5}{|l|}{ Incarcerated hernia } \\
\hline No & $71(68.9)$ & $30(76.9)$ & $41(64.1)$ & \multirow[t]{3}{*}{0.1713} \\
\hline Yes & $32(31.1)$ & $9(23.1)$ & $23(35.1)$ & \\
\hline Missing data & 2 & 0 & 2 & \\
\hline Hernia defect width $(\mathrm{cm})$ & $3.9(2.5)$ & $2.3(1.0)$ & $4.9(2.6)$ & $<0.0001$ \\
\hline Missing data & 1 & 0 & 1 & \\
\hline Hernia defect length $(\mathrm{cm})$ & $5.0(4.1)$ & $2.5(1.2)$ & $6.5(4.5)$ & $<0.0001$ \\
\hline Missing data & 1 & 0 & 1 & \\
\hline Hernia defect area $\left(\mathrm{cm}^{2}\right)$ & $21.9(33.3)$ & $5.2(5.6)$ & $31.9(38.7)$ & $<0.0001$ \\
\hline Missing data & 1 & 0 & 1 & \\
\hline Mesh positioning time (minutes) & $9.4(6.3)$ & $8.5(5.6)$ & $9.9(6.6)$ & 0.2652 \\
\hline Missing data & 3 & 1 & 2 & \\
\hline Mesh area to defect area ratio $(M / D)$ & $35.1(46.4)$ & $55.7(64.1)$ & $22.7(24.8)$ & $<0.0001$ \\
\hline Missing data & 1 & 0 & 1 & \\
\hline
\end{tabular}

Results are expressed in $\mathrm{n}(\%)$ for categorical data and mean (SD) for quantitative data; $p$ value $<0.05$ is significant
(22 cases) tackers or with sutures in 11 cases. Double crown stapling was used in 17 cases. Glue was not used. Mesh overlap $\geq 5 \mathrm{~cm}$ was achieved in 76 of 80 laparoscopic repairs (95.0\%; excludes hybrid repairs) and 16 of 22 open repairs $(72.7 \%)$.

\section{Mesh area-to-defect area ratios}

Information on $M / D$ ratios was collected in 104 of 105 hernias (Table 3 ). The mean $M / D$ ratio was 55.7 ( \pm 64.1 ) and $22.7( \pm 24.8)$ for primary and incisional hernia defects, respectively $(p<0.0001)$. The ratio was larger for laparoscopic repairs than open repairs $(39.9 \pm 49.0$ vs. $12.0 \pm 15.4$, $p<0.0001$; combined open/laparoscopic 77.1 \pm 75.1 ).

\section{Symbotex composite mesh handling characteristics}

Flexibility of the mesh and ease of insertion were rated as "satisfying" in 102 hernia repairs (Tables 5, 6). Similarly, in 80 hernias, the mesh was reported as "satisfying" to trim (not applicable or unknown $n=20$ ). Shape memory was also generally considered "satisfying," as was the low visceral attachment. In two hernia repairs, shape memory was rated as "unsatisfying" (2.1\%). Several features of the mesh were identified as beneficial to successfully completing operations (Tables 5, 7).

\section{Follow-up and adverse events}

Patients underwent follow-up for a mean of 719.2 days (23.9 months; Fig. 1). There were no deaths nor serious adverse events reported during this study (available patient data at end of year $1 n=94$, year $2 n=93$ ).

In total, ten adverse events (primary hernia $4 / 38$ patients, $10.5 \%$; incisional hernia $6 / 62,9.7 \%$ ) were reported in the course of 24-month follow-up. During another surgical procedure, one sub-costal incisional hernia was noted 6-12 months after midline incisional hernia repair using SCM. Though likely to be a new hernia following previous hepatic surgery, this defect was classified as a "recurrence". According to the operating surgeon, the small, asymptomatic defect does not at present require a reintervention. 
Table 3 Summary of operative data by hernia type, including whether an emergency or elective procedure, Altemeier classification, type of anesthesia administered, surgical approach, use of antibiotic prophylaxis, total operating time and time for mesh placement, the mesh/ defect ration $(M / D)$ and hospital stay $(n=100)$

\begin{tabular}{|c|c|c|c|c|}
\hline Criteria & $\begin{array}{l}\text { Overall patients } \\
(n=100)\end{array}$ & $\begin{array}{l}\text { Primary hernias } \\
\text { patients }(n=38)\end{array}$ & $\begin{array}{l}\text { Incisional hernias } \\
\text { patients }(n=62)\end{array}$ & $p$ value \\
\hline \multicolumn{5}{|l|}{ Emergency surgery } \\
\hline No & $97(97.0)$ & 37 (97.4) & $60(96.8)$ & \multirow[t]{2}{*}{1.0000} \\
\hline Yes & $3(3.0)$ & $1(2.6)$ & $2(3.2)$ & \\
\hline \multicolumn{5}{|l|}{ Altemeier classification } \\
\hline Clean & $97(97.0)$ & 37 (97.4) (89.5) & $60(96.8)$ & \multirow[t]{2}{*}{1.0000} \\
\hline Clean contaminated & $3(3.0)$ & $1(2.6)$ & $2(3.2)$ & \\
\hline \multicolumn{5}{|l|}{ Surgical approach } \\
\hline Open (laparotomy) & $20(20.0)$ & $0(0.0)$ & $20(32.3)$ & \multirow[t]{3}{*}{$<0.0001$} \\
\hline Laparoscopic & $77(77.0)$ & $37(97.4)$ & $40(64.5)$ & \\
\hline Hybrid open/laparoscopic & $3(3.0)$ & $1(2.6)$ & $2(3.2)$ & \\
\hline \multicolumn{5}{|l|}{ Antibiotic prophylaxis } \\
\hline No & $15(15.0)$ & $11(28.9)$ & $4(6.5)$ & \multirow[t]{4}{*}{0.0022} \\
\hline Yes & $85(85.0)$ & $27(71.1)$ & $58(93.5)$ & \\
\hline Single dose & $84(84.0)$ & $27(71.1)$ & $57(91.9)$ & \\
\hline Prolonged & $1(1.0)$ & $0(0.0)$ & $1(1.6)$ & \\
\hline Operative time (min) & $43.4(27.3)$ & $30.9(21.8)$ & $50.9(27.7)$ & 0.0004 \\
\hline Missing data & 4 & 2 & 2 & \\
\hline Hospital stay (days) & $2.5(2.3)$ & $0.9(1.1)$ & $2.9(2.1)$ & $<0.0001$ \\
\hline Missing data & 8 & 2 & 6 & \\
\hline
\end{tabular}

Results are expressed in $n(\%)$ for categorical data and mean (SD) for quantitative data; $p$ value $<0.05$ is significant

Table 4 Summary of operative data by surgical approach (laparoscopic, open, combined open/laparoscopic): total operating time, time for mesh placement and hospital stay $(n=100)$

\begin{tabular}{|c|c|c|c|c|}
\hline Criteria & $\begin{array}{l}\text { Laparoscopic approach } \\
\text { patients }(n=77)\end{array}$ & $\begin{array}{l}\text { Open approach } \\
\text { patients }(n=20)\end{array}$ & $\begin{array}{l}\text { Hybrid open/lap approach } \\
\text { patients }(n=3)\end{array}$ & $p$ value \\
\hline \multicolumn{5}{|l|}{ Hernia type } \\
\hline Primary hernia & $37(48.1)$ & $0(0.0)$ & $1(33.3)$ & $<0.0001$ \\
\hline Incisional hernia & $40(51.9)$ & $20(100.0)$ & $2(66.7)$ & \\
\hline Operative time (min) & $36.2(23.5)$ & $67.4(25.8)$ & $68.3(32.1)$ & $<0.0001$ \\
\hline Missing data & 3 & 1 & 0 & \\
\hline Operative time for primary hernia patients & $30.3(21.7)$ & $\mathrm{n} / \mathrm{a}$ & $55(0.0)$ & $\mathrm{n} / \mathrm{a}$ \\
\hline Missing data & 2 & 0 & 0 & \\
\hline Operative time for Incisional hernia patients & $41.6(24.0)$ & $67.4(25.8)$ & $75.0(42.4)$ & $<0.0001$ \\
\hline Missing data & 0 & 1 & 0 & \\
\hline Hospital stay (days) & $1.6(1.6)$ & $4.7(2.0)$ & $2.9(2.1)$ & $<0.0001$ \\
\hline Missing data & 5 & 3 & 0 & \\
\hline
\end{tabular}

Results are expressed in $\mathrm{n}(\%)$ for categorical data and mean (SD) for quantitative data; $p$ value $<0.05$ is significant $p$ values are for laparoscopic vs. open approach

Three patients experienced a mild, transitory ileus after incisional hernia repair (peri-operative $2 / 100 ; 2-4$ weeks post-operative 1/82). It is unknown whether these experiences were related to intra-operative adhesiolysis, mesh placement or the implant itself. Similarly, six patients were noted to have had a seroma (peri-operative 1/100;
2-4 weeks post-operative $5 / 82,6.1 \%$ ). In four, the seroma followed a primary hernia repair, and in two, an incisional hernia repair. These seromas were not considered mesh related. One seroma required aspiration, whereas all others resolved spontaneously. 
Table 5 Summary of the defect size and $M / D$ ratio according to fascial closure and surgical approach

\begin{tabular}{|c|c|c|c|c|}
\hline \multirow[t]{2}{*}{ Overall hernias ( $n=105$ hernias) } & \multicolumn{2}{|c|}{ Laparoscopic approach } & \multicolumn{2}{|l|}{ Open approach } \\
\hline & $\begin{array}{l}\text { Closed fascia } \\
(n=26 \text { hernias })\end{array}$ & $\begin{array}{l}\text { Unclosed fascia } \\
(n=57 \text { hernias })\end{array}$ & Closed fascia ( $n=9$ hernias) & $\begin{array}{l}\text { Unclosed fascia } \\
(n=13 \text { hernias })\end{array}$ \\
\hline Defect area $\left(\mathrm{cm}^{2}\right)$ & $16.4(12.1)$ & $9.7(17.5)$ & $61.5(61.5)$ & $58.1(43.9)$ \\
\hline Missing data & 0 & 1 & 0 & 0 \\
\hline$p$ value (closed vs. unclosed) & 0.0009 & & 0.6159 & \\
\hline Ratio mesh size/defect area & $25.4(22.9)$ & $48.7(57.1)$ & $19.7(22.3)$ & $6.7(3.1)$ \\
\hline Missing data & 0 & 1 & 0 & 0 \\
\hline$p$ value (closed vs. unclosed) & 0.0018 & & 0.2699 & \\
\hline Primary hernias ( $n=39$ hernias) & $\begin{array}{l}\text { Closed fascia } \\
(n=10 \text { her- } \\
\text { nias })\end{array}$ & $\begin{array}{l}\text { nclosed fascia } \\
\imath=29 \text { hernias) }\end{array}$ & sed fascia $(n=0)$ & sed fascia $(n=0)$ \\
\hline Defect area $\left(\mathrm{cm}^{2}\right)$ & $8.5(9.4)$ & $1(3.0)$ & (primary hernias were all repaired laparoscopically) & \\
\hline Missing data & 0 & & & \\
\hline$p$ value (closed vs. unclosed) & 0.1456 & & & \\
\hline Ratio mesh size/defect area & $39.5(28.7)$ & $1.4(72.0)$ & & \\
\hline Missing data & 0 & & & \\
\hline$p$ value (closed vs. unclosed) & 0.4076 & & & \\
\hline $\begin{array}{l}\text { Results for incisional hernias } \\
(n=66 \text { hernias })\end{array}$ & $\begin{array}{l}\text { Closed fascia } \\
(n=16 \text { hernias })\end{array}$ & $\begin{array}{l}\text { Unclosed fascia } \\
(n=28 \text { hernias })\end{array}$ & Closed fascia ( $n=9$ hernias) & $\begin{array}{l}\text { Unclosed fascia } \\
(n=13 \text { hernias })\end{array}$ \\
\hline Defect area $\left(\mathrm{cm}^{2}\right)$ & $21.3(11.2)$ & $15.7(23.8)$ & $61.5(61.5)$ & $58.1(43.9)$ \\
\hline Missing data & 0 & 1 & 0 & 0 \\
\hline$p$ value (closed vs. unclosed) & 0.0071 & & 0.6159 & \\
\hline Ratio mesh size/defect area & $16.6(12.8)$ & $35.0(30.8)$ & $19.7(22.3)$ & $6.7(3.1)$ \\
\hline Missing data & 0 & 1 & 0 & 0 \\
\hline$p$ value (closed vs. unclosed) & 0.0055 & & 0.2699 & \\
\hline
\end{tabular}

\section{Patient satisfaction}

At 12 months after surgery, 94 patients took part in a selfadministered questionnaire. At 24 months, 93 patients were responding to the questionnaire. Earlier research demonstrated that post-operative pain scores improved progressively with time [14]. At 12 months, patients' average VAS score was 3; by the second year, this had reduced to 2 .

Patient satisfaction is shown in Table 8. The proportion of patients rating their outcome as "good" or "excellent" rose slightly with time (year $188.3 \%$ vs. year $294.5 \%$ ) while the proportion considering their outcome "medium" or "bad" fell from $11.7 \%$ at year 1 to $5.5 \%$ at year 2. There was no significant difference between ratings by patients undergoing primary and incisional hernia repair.

At year 1, 90 of 94 (95.7\%) considered their abdominal wall "firm," compared with 91 of 93 (97.8\%) at year 2 (Table 8). Of 93 patients, 6 (all in the incisional hernia group) reported a lump at year 1 , compared with 2 (both in the incisional hernia group) at year 2 .

In response to the question "Do you feel pain or discomfort?", 71 of 94 patients said "No" (75.5\%) at year 1, compared with 81 of $93(87.1 \%)$ at year 2 . Of the 12 patients (12.9\%) with symptoms at year 2, 5 (5.4\%) described these symptoms as "discomfort," $6(6.5 \%)$ as "moderate pain not requiring analgesia", and $1(1.1 \%)$ as "loss of sensitivity." Further details are provided in Table 8 .

\section{Discussion}

The results of this observational study of 100 patients extracted from the Club Hernie registry show that patients with a primary or incisional ventral hernia generally experienced discomfort/pain or dysesthesia before surgery. Followup at 24 months after a laparoscopic or open repair found that nearly $90 \%$ of patients were pain and discomfort free, and almost $100 \%$ were unable to feel a lump; most grading their result as 'good' or 'excellent'.

Using SCM to repair primary and incisional abdominal hernias was found to be safe and effective. Early postoperative complications were mild (Clavien-Dindo $\leq 2$ ) and non-life threatening: [22]. There was one suspected hernia recurrence detected during another procedure 6-12 
Table 6 Surgeons' views of Symbotex mesh handling characteristics: mesh flexibility, ease of mesh trimming, ease of mesh insertion, mesh shape memory and low visceral attachment

Table 7 Features of Symbotex mesh found by surgeons to assist primary and incisional hernia repair

\begin{tabular}{|c|c|c|c|c|}
\hline Criteria & $\begin{array}{l}\text { Overall hernias } \\
(n=105)\end{array}$ & $\begin{array}{l}\text { Primary hernias } \\
(n=39)\end{array}$ & $\begin{array}{l}\text { Incisional hernias } \\
(n=66)\end{array}$ & $p$ value \\
\hline \multicolumn{5}{|l|}{ Mesh flexibility } \\
\hline Satisfying & $102(100.0)$ & $38(100.0)$ & $64(100.0)$ & \multirow[t]{4}{*}{1.0000} \\
\hline Unsatisfying & $0(0.0)$ & $0(0.0)$ & $0(0.0)$ & \\
\hline $\mathrm{n} / \mathrm{a}$ & 0 & 0 & 0 & \\
\hline Missing data & 3 & 1 & 2 & \\
\hline \multicolumn{5}{|c|}{ Ease of mesh trimming } \\
\hline Satisfying & $80(100.0)$ & $31(100.0)$ & $49(100.0)$ & \multirow[t]{4}{*}{1.0000} \\
\hline Unsatisfying & $0(0.0)$ & $0(0.0)$ & $0(0.0)$ & \\
\hline $\mathrm{n} / \mathrm{a}$ & 20 & 6 & 14 & \\
\hline Missing data & 5 & 2 & 3 & \\
\hline \multicolumn{5}{|c|}{ Ease of mesh insertion } \\
\hline Satisfying & $102(100.0)$ & $38(100.0)$ & $64(100.0)$ & \multirow[t]{4}{*}{1.0000} \\
\hline Unsatisfying & $0(0.0)$ & $0(0.0)$ & $0(0.0)$ & \\
\hline $\mathrm{n} / \mathrm{a}$ & 0 & 0 & 0 & \\
\hline Missing data & 5 & 1 & 3 & \\
\hline \multicolumn{5}{|c|}{ Mesh shape memory } \\
\hline Satisfying & $94(97.9)$ & $36(100.0)$ & $58(96.7)$ & \multirow[t]{4}{*}{0.5260} \\
\hline Unsatisfying & $2(2.1)$ & 0 & $2(3.3)$ & \\
\hline $\mathrm{n} / \mathrm{a}$ & 2 & 0 & 2 & \\
\hline Missing data & 7 & 3 & 4 & \\
\hline \multicolumn{5}{|c|}{ Low visceral attachment } \\
\hline Satisfying & $92(100.0)$ & $35(100.0)$ & $57(100.0)$ & \multirow[t]{4}{*}{1.0000} \\
\hline Unsatisfying & $0(0.0)$ & $0(0.0)$ & $0(0.0)$ & \\
\hline $\mathrm{n} / \mathrm{a}$ & 9 & 2 & 7 & \\
\hline Missing data & 4 & 2 & 2 & \\
\hline
\end{tabular}

Results are expressed in $n(\%) ; p$ value $<0.05$ is significant

$n / a$ is for "not-applicable" (surgeon did not have opportunity to evaluate the mesh property)

\begin{tabular}{lllll}
\hline Criteria & $\begin{array}{l}\text { Overall her- } \\
\text { nias }(n=105)\end{array}$ & $\begin{array}{l}\text { Primary her- } \\
\text { nias }(n=39)\end{array}$ & $\begin{array}{l}\text { Incisional her- } \\
\text { nias }(n=66)\end{array}$ & $p$ value \\
\hline Mesh transparency aids placement & $74(74.0)$ & $26(72.2)$ & $48(75.0)$ & 0.7610 \\
Missing data & 5 & 3 & 2 & \\
Mesh marking assists placement & $68(68.0)$ & $28(77.8)$ & $40(62.5)$ & 0.1160 \\
Missing data & 5 & 3 & 2 & \\
Prosthesis shape memory assists placement & $53(53.0)$ & $14(38.9)$ & $39(60.9)$ & 0.0340 \\
Missing data & 5 & 3 & 2 & 0.3250 \\
Mesh easy to reposition & $51(51.0)$ & $16(44.4)$ & $35(54.7)$ & 2 \\
Missing data & 5 & 3 & $29(45.3)$ & 0.5330 \\
Mesh does not switch during fixation/stapling & $43(43.0)$ & $14(38.9)$ & 2 & \\
Missing data & 5 & 3 & $18(28.1)$ & 0.0980 \\
Mesh conformability to the anatomy during & $34(34.0)$ & $16(44.4)$ & & \\
placement is useful & & 3 & $16(25.0)$ & 0.0460 \\
Missing data & 5 & $16(44.4)$ & 2 & \\
No requirement to orient mesh is useful & $32(32.0)$ & 3 & & 2 \\
Missing data & 5 & & & \\
\hline
\end{tabular}

Results are expressed in $n(\%) ; p$ value $<0.05$ is significant 
Table 8 Patient-related outcome measures (PROM) at the end of year 1 and year 2: overall satisfaction, perceived abdominal wall firmness, pain/discomfort, location of any symptoms, timing of symptoms, fre- quency of symptoms, effect of symptoms on activity and comparison of symptom nuisance before and after surgery

\begin{tabular}{|c|c|c|c|c|c|c|c|c|}
\hline \multirow[t]{2}{*}{ Criteria } & \multicolumn{4}{|c|}{ Response at 1 year } & \multicolumn{4}{|c|}{ Response at 2 year } \\
\hline & $\begin{array}{l}\text { Overall } \\
\text { patients } \\
(n=100)\end{array}$ & $\begin{array}{l}\text { Primary hernias } \\
\text { patients }(n=38)\end{array}$ & $\begin{array}{l}\text { Incisional } \\
\text { hernias patients } \\
(n=62)\end{array}$ & $p$ value & $\begin{array}{l}\text { Overall } \\
\text { patients } \\
(n=100)\end{array}$ & $\begin{array}{l}\text { Primary hernias } \\
\text { patients }(n=38)\end{array}$ & $\begin{array}{l}\text { Incisional } \\
\text { hernias patients } \\
(n=62)\end{array}$ & $p$ value \\
\hline \multicolumn{9}{|l|}{$\begin{array}{l}\text { Q1: Does your } \\
\text { abdominal wall } \\
\text { seem firm? }\end{array}$} \\
\hline No & $4(4.3)$ & $0(0.0)$ & $4(6.9)$ & 0.2942 & $2(2.2)$ & $0(0.0)$ & $2(3.4)$ & 0.5255 \\
\hline Yes & $90(95.7)$ & $36(100.0)$ & $54(93.1)$ & & $91(97.8)$ & $35(100.0)$ & $56(96.6)$ & \\
\hline Missing data & 6 & 2 & 4 & & 7 & 3 & 4 & \\
\hline \multicolumn{9}{|l|}{$\begin{array}{l}\text { Q2: Do you feel a } \\
\text { lump? }\end{array}$} \\
\hline No & $87(93.5)$ & $36(100.0)$ & $51(89.5)$ & 0.0780 & $91(97.8)$ & $35(100.0)$ & $56(96.6)$ & 0.5250 \\
\hline Yes & $6(6.5)$ & $0(0.0)$ & $6(10.5)$ & & $2(2.2)$ & $0(0.0)$ & $2(3.4)$ & \\
\hline $\begin{array}{l}\text { Yes, on operated } \\
\text { area }\end{array}$ & $2(2.2)$ & $0(0.0)$ & $2(3.5)$ & & $0(0.0)$ & $0(0.0)$ & $0(0.0)$ & \\
\hline Yes, on midline area & $3(3.2)$ & $0(0.0)$ & $3(5.2)$ & & $2(2.2)$ & $0(0.0)$ & $2(3.4)$ & \\
\hline Yes, elsewhere & $1(1.1)$ & $0(0.0)$ & $1(1.7)$ & & $0(0.0)$ & $0(0.0)$ & $0(0.0)$ & \\
\hline Missing data & 7 & 2 & 5 & & 7 & 3 & 4 & \\
\hline \multicolumn{9}{|l|}{$\begin{array}{l}\text { Q3: Do you feel any } \\
\text { pain or discomfort? }\end{array}$} \\
\hline No & $71(75.5)$ & $29(80.6)$ & $42(72.4)$ & 0.3560 & $81(87.1)$ & $32(91.4)$ & $49(84.5)$ & 0.3470 \\
\hline Yes & $23(24.5)$ & $7(19.4)$ & $16(27.6)$ & & 12 (12.9) & $3(8.6)$ & $9(15.5)$ & \\
\hline $\begin{array}{l}\text { Yes, mild pain or } \\
\text { discomfort }\end{array}$ & $13(13.8)$ & $6(16.7)$ & $7(12.1)$ & & $5(5.4)$ & $1(2.9)$ & $4(6.9)$ & \\
\hline $\begin{array}{l}\text { Yes, moderate pain } \\
\text { (no analgesia } \\
\text { required) }\end{array}$ & $8(8.5)$ & $1(2.8)$ & $7(12.1)$ & & $6(6.5)$ & $2(5.7)$ & $4(6.9)$ & \\
\hline $\begin{array}{l}\text { Yes, severe pain } \\
\text { (analgesia } \\
\text { required) }\end{array}$ & $1(1.1)$ & $0(0.0)$ & $1(1.7)$ & & $0(0.0)$ & $0(0.0)$ & $0(0.0)$ & \\
\hline $\begin{array}{l}\text { Yes, loss of sensitiv- } \\
\text { ity }\end{array}$ & $0(0.0)$ & $0(0.0)$ & $0(0.0)$ & & $1(1.1)$ & $0(0.0)$ & $1(1.7)$ & \\
\hline $\begin{array}{l}\text { Yes, other (descrip- } \\
\text { tion is missing) }\end{array}$ & $1(1.1)$ & $0(0.0)$ & $1(1.7)$ & & $0(0.0)$ & $0(0.0)$ & $0(0.0)$ & \\
\hline Missing data & 6 & 2 & 4 & & 7 & 3 & 4 & \\
\hline \multicolumn{9}{|l|}{$\begin{array}{l}\text { Q4 through Q8 are } \\
\text { restricted to patients } \\
\text { feeling pain or } \\
\text { discomfort }\end{array}$} \\
\hline \multicolumn{9}{|l|}{$\begin{array}{l}\text { Q4: Where are the } \\
\text { symptoms located? }\end{array}$} \\
\hline Operated hernia & $5(21.7)$ & $2(28.6)$ & $3(18.8)$ & & $0(0.0)$ & $0(0.0)$ & $0(0.0)$ & \\
\hline Midline & $17(73.9)$ & $5(71.4)$ & $12(75.0)$ & & $11(91.7)$ & $2(66.7)$ & $9(100.0)$ & \\
\hline Controlateral & $1(4.3)$ & $0(0.0)$ & $1(6.3)$ & & $0(0.0)$ & $0(0.0)$ & $0(0.0)$ & \\
\hline Testicle region & $0(0.0)$ & $0(0.0)$ & $0(0.0)$ & & $1(8.3)$ & $1(33.3)$ & $0(0.0)$ & \\
\hline Missing data & 0 & 0 & 0 & & 0 & 0 & 0 & \\
\hline \multicolumn{9}{|l|}{$\begin{array}{l}\text { Q5: When do you feel } \\
\text { these symptoms? }\end{array}$} \\
\hline $\begin{array}{l}\text { During lifting, } \\
\text { coughing }\end{array}$ & $0(0.0)$ & $0(0.0)$ & $0(0.0)$ & & $0(0.0)$ & $0(0.0)$ & $0(0.0)$ & \\
\hline $\begin{array}{l}\text { During other types } \\
\text { of efforts }\end{array}$ & $11(50.0)$ & $2(33.3)$ & $9(56.3)$ & & $3(30.0)$ & $1(50.0)$ & $2(25.0)$ & \\
\hline
\end{tabular}


Table 8 (continued)

\begin{tabular}{|c|c|c|c|c|c|c|c|c|}
\hline \multirow[t]{2}{*}{ Criteria } & \multicolumn{4}{|c|}{ Response at 1 year } & \multicolumn{4}{|c|}{ Response at 2 year } \\
\hline & $\begin{array}{l}\text { Overall } \\
\text { patients } \\
(n=100)\end{array}$ & $\begin{array}{l}\text { Primary hernias } \\
\text { patients }(n=38)\end{array}$ & $\begin{array}{l}\text { Incisional } \\
\text { hernias patients } \\
(n=62)\end{array}$ & $p$ value & $\begin{array}{l}\text { Overall } \\
\text { patients } \\
(n=100)\end{array}$ & $\begin{array}{l}\text { Primary hernias } \\
\text { patients }(n=38)\end{array}$ & $\begin{array}{l}\text { Incisional } \\
\text { hernias patients } \\
(n=62)\end{array}$ & $p$ value \\
\hline At the end of the day & $1(4.5)$ & $1(16.7)$ & $0(0.0)$ & & $0(0.0)$ & $0(0.0)$ & $0(0.0)$ & \\
\hline At any time & $10(45.5)$ & $3(50.0)$ & $7(43.8)$ & & $7(70.0)$ & $1(50.0)$ & $6(75.0)$ & \\
\hline Missing data & 1 & 1 & 0 & & 2 & 1 & 1 & \\
\hline \multicolumn{9}{|l|}{$\begin{array}{l}\text { Q6: How often do you } \\
\text { feel these symp- } \\
\text { toms? }\end{array}$} \\
\hline Rarely & $8(38.1)$ & $2(33.3)$ & $6(40.0)$ & & $5(50.0)$ & $1(50.0)$ & $4(50.0)$ & \\
\hline Several times a week & $9(42.9)$ & $3(50.0)$ & $6(40.0)$ & & $1(10.0)$ & $0(0.0)$ & $1(12.5)$ & \\
\hline Several times a day & $1(4.8)$ & $0(0.0)$ & $1(6.7)$ & & $2(20.0)$ & $1(50.0)$ & $1(12.5)$ & \\
\hline Often & $1(4.8)$ & $0(0.0)$ & $1(6.7)$ & & $2(20.0)$ & $0(0.0)$ & $2(25.0)$ & \\
\hline $\begin{array}{l}\text { Thoughout the day/ } \\
\text { all day long }\end{array}$ & $2(9.5)$ & $1(16.7)$ & $1(6.7)$ & & $0(0.0)$ & $0(0.0)$ & $0(0.0)$ & \\
\hline Missing data & 2 & 1 & 1 & & 2 & 1 & 1 & \\
\hline \multicolumn{9}{|l|}{$\begin{array}{l}\text { Q7: Effect of symp- } \\
\text { toms on activity }\end{array}$} \\
\hline $\begin{array}{l}\text { Do not hinder your } \\
\text { activities }\end{array}$ & $14(66.7)$ & $4(66.7)$ & $10(66.7)$ & & $7(70.0)$ & $1(50.0)$ & $6(75.0)$ & \\
\hline $\begin{array}{l}\text { Allow you to pursue } \\
\text { activities at a } \\
\text { slower pace }\end{array}$ & $7(33.3)$ & $2(33.3)$ & $5(33.3)$ & & $3(30.0)$ & $1(50.0)$ & $2(25.0)$ & \\
\hline $\begin{array}{l}\text { Cause temporary } \\
\text { interruption of } \\
\text { your activities }\end{array}$ & $0(0.0)$ & $0(0.0)$ & $0(0.0)$ & & $0(0.0)$ & $0(0.0)$ & $0(0.0)$ & \\
\hline $\begin{array}{l}\text { Prevent certain } \\
\text { activities }\end{array}$ & $0(0.0)$ & $0(0.0)$ & $0(0.0)$ & & $0(0.0)$ & $0(0.0)$ & $0(0.0)$ & \\
\hline Missing data & 2 & 1 & 1 & & 2 & 1 & 1 & \\
\hline \multicolumn{9}{|l|}{$\begin{array}{l}\text { Q8: Nuisance of pain/ } \\
\text { discomfort is }\end{array}$} \\
\hline $\begin{array}{l}<\text { Hernia discomfort } \\
\text { before surgery }\end{array}$ & $18(81.8)$ & $5(71.4)$ & $13(86.7)$ & & 7 (63.6) & $2(66.7)$ & $5(62.5)$ & \\
\hline $\begin{array}{l}>\text { Hernia discomfort } \\
\text { before surgery }\end{array}$ & $4(18.2)$ & $2(28.6)$ & $2(13.3)$ & & $4(36.4)$ & $1(33.3)$ & $3(37.5)$ & \\
\hline Missing data & 1 & 0 & 1 & & 1 & 0 & 1 & \\
\hline \multicolumn{9}{|l|}{$\begin{array}{l}\text { Q9: Patient satisfac- } \\
\text { tion }\end{array}$} \\
\hline Excellent & $10(10.6)$ & $6(16.7)$ & $4(6.9)$ & 0.5262 & $7(7.7)$ & $1(2.9)$ & $6(10.5)$ & 0.2693 \\
\hline Good & 73 (77.7) & $27(75.0)$ & $46(79.3)$ & & $79(86.8)$ & $32(94.1)$ & $47(82.5)$ & \\
\hline Medium & $7(7.4)$ & $2(5.6)$ & $5(8.6)$ & & $3(3.3)$ & $0(0.0)$ & $3(5.3)$ & \\
\hline $\mathrm{Bad}$ & $4(4.3)$ & $1(2.8)$ & $3(5.2)$ & & $2(2.2)$ & $1(2.9)$ & $1(1.8)$ & \\
\hline Missing data & 6 & 2 & 4 & & 9 & 4 & 5 & \\
\hline
\end{tabular}

Results are expressed in $\mathrm{n}(\%) ; p$ value $<0.05$ is significant

months after surgery which is not at present considered to require treatment. On inspection, this was found to be a sub-costal defect which was not originally treated in the study. Notably, the study population was unselected, generally middle aged or elderly, and overweight, and had risk factors potentially complicating surgical dissection and post-operative recovery. Surgeons reported that the mesh generally handled to their satisfaction during both laparoscopic and open approaches.

Hauters et al. stated that "The ultimate measure of hernia repair remains its recurrence rate" [10]. Nearly twothirds of hernias in the present study were classified as incisional. Whereas meta-analyses of laparoscopic and open incisional ventral hernia repairs in 1,003 patients 
have shown recurrence rates of 6.99 and $4.82 \%$, respectively [23], Chelala et al. have suggested that the true recurrence following laparoscopic repair of wider defects is around $12 \%$, with values after open repair in exceptional circumstances reaching $32 \%$ [13]. This study's early finding of only one possible hernia recurrence in a complex patient who had undergone previous liver surgery is encouraging but needs to be put into context. Patients were not examined at 24 months, nor did they undergo objective tests such as abdominal ultrasound scanning. As hernias can recur at any point during follow-up, later defects may be discovered in other patients in the present cohort $[4,5$, 23]. However, later hernias become increasingly unlikely with time-most hernias present within 24 months of surgery, with a mean delay of $19 \pm 13$ months [10].

Early post-operative complications are important as they may necessitate further treatment and delay discharge. In a prospective cohort study of 4565 patients, Kroese et al. reported an overall 30-day complication rate of $4.4 \%$ following primary ventral hernia repair, compared with $15 \%$ for incisional hernias $(p<0.001)$ [16]. In the present study, nine patients experienced an early seroma or ileus with no clear relation to the hernia type or use of mesh. All resolved spontaneously except for one seroma, which required aspiration. There were no wound infections. Other researchers have reported a number of major complications [13].

Although a restored QoL free from abdominal wall symptoms may be as key to procedural success as a low hernia recurrence rate for many patients, this priority has been often ignored in past research [24]. By comparison, our study focused on the PROM concept. We found that $94.5 \%$ of patients rated their outcome as "good" or "excellent" at year 2, a slight improvement from year $1(88.3 \%)$, while patients considering their outcome "medium" or "bad" fell between years 1 and $2(11.7 \%$ vs. $5.5 \%)$, and those considering their abdominal wall to be "firm" increased from 95.7 to $97.8 \%$. Of 93 patients, 6 reported a lump at 12 months, and 2 reported a lump at 24 months, all in the incisional hernia group. Both lumps were in the midline and not located at the original site hernia site.

The high satisfaction rate in this study is probably related to patients' low hernia recurrence rate and limited post-operative pain. Satisfaction did not appear to be influenced by the experiences of 12 patients reporting generally minor symptoms with no or limited effect on activity at 2 years. Liang et al. reported that $74.5 \%$ of patients were satisfied with the outcome of their laparoscopic hernia repair, and that chronic pain, changes in functional status, and recurrence were associated with reduced overall satisfaction [25]. Langbach et al. confirmed that results could be predicted by the absence of chronic pain and recurrence [26]. In the latter study, only $60.5 \%$ of patients were satisfied with their outcome after a mean of 48 months following laparoscopic ventral hernia repair, compared with $49.3 \%$ after open repair at 52 months.

Several operative factors may have contributed to the medium-term results encountered in the present study's heterogeneous population. Guerin and Turquier previously demonstrated that the stresses applied to a piece of implanted mesh used to repair a ventral hernia are influenced by defect size and the degree of overlap with the surrounding abdominal wall [27]. Leblanc empirically recommends that there should be at least $5 \mathrm{~cm}$ of overlap in all directions [9]. These factors were examined further by Hauters et al., who concluded that while overlap is important, the $M / D$ ratio is the only independent predictor of recurrence [10]. Among 16 recurrences in a cohort of 213 laparoscopic primary ventral or incisional hernia repair patients, the authors reported $70 \%, 35 \%, 9 \%$, and $0 \%$ failure rates when the mean $M / D$ ratio was $\leq 8$, between 9 and 12 , between 13 and 16 , and $\geq 17$, respectively.

It should be noted in the above study that the authors used a bridging technique without closing the defect. Baker et al., in a nationwide cohort from Denmark recently showed a reduced risk of reoperation for ventral hernia recurrences if the defect is closed during laparoscopic repair, in addition to the use of permanent tacks [28]. Though not universally recorded in the present study, the use of fascial closure may have contributed to the low recurrence rate to date, along with greater confidence among Club Hernie surgeons to use large pieces of mesh even for mid-sized defects. The $M / D$ ratios of 55.7 and 22.7 for primary and incisional hernia defects in the present study are clearly larger than those reported by others [10].

Choice of mesh can influence outcomes. Liang et al. reported that polypropylene and polytetrafluoroethylene were associated with higher rates of chronic pain [25]. By comparison, implantation of low-density mesh may result in more recurrences. A multi-institutional study found that midweight mesh produced less surgical site infections and shorter hospital stays, and was associated with higher QoL scores at 6-12 months than lightweight mesh [29].

$\mathrm{SCM}$, the successor to PCO, was developed with the intention of fulfilling many of the characteristics of an ideal mesh [30]. At $64 \mathrm{~g} / \mathrm{m}^{2}$, it is classified as midweight and has a monofilament structure designed to encourage tissue ingrowth and mesh fixation. This feature may also reduce the risk of infection. Because SCM is relatively thin $(0.7 \mathrm{~mm})$, it provides flexibility, good shape memory, and ease of insertion and deployment-features of importance when using the IPOM technique. While the risk of adhesion formation has not been assessed in this study, the fact that SCM has a continuous hydrophilic film similar to PCO's suggests that it is likely to have equivalent performances. An ultrasound examination of wound sites 12 months after PCO placement showed that $86 \%$ of patients 
were free of adhesions following incisional and umbilical hernia repair [12]. The success of this film was further demonstrated in a study by Chelala et al. [13]. As part of a long-term follow-up series on 1326 patients, 126 underwent further abdominal surgery for a variety of reasons, affording the opportunity to inspect for adhesions: $45.2 \%$ were adhesion free, $42.1 \%$ had loose omental adhesions, and $12.7 \%$ had easily cleavable or mild serosal adhesions to the PCO mesh.

All surgeons in this study reported that they were satisfied with the mesh's flexibility, ease of trimming, and easy of insertion. Similarly, most were satisfied with its shape memory and low visceral attachment. Among the features that were highlighted as assisting placement were mesh transparency (74\%) and orientation marking (68\%). SCM appeared to be equally suitable for use in open or laparoscopic procedures, with no significant differences between approaches in the time required to position the mesh.

The strengths of the present study included an unselected cohort information being collected from both surgeons and patients from different centers, and the use of a PROM concept. Data taken from consecutive repairs of both primary and incisional hernias with either operative approach and no limitation on manner of fixation or closure of the defect represent the real-world approach of active surgeons. Further, to date there are few published studies using SCM for hernia repair.

This study did, however, have some limitations: numbers were small, there was no comparator group, patients were not physically examined at 24 months, and no objective tests were performed to assess the abdominal wall for complications. While the questionnaire is considered reliable and comparable with practices in other registries, some complications may have been missed [16, 31]. The questionnaire also screened for further repairs (Table 8); however, evidence from Helgstand et al., has shown that the reoperation rate underestimates the overall risk of recurrence by four- to fivefold [32]. This view is at odds with Hauters et al,, who used a phone interview to study outcomes among 213 patients and consider this approach sufficient for detecting recurrences accurately. They make the point that, as all patients in their study had previously experienced a hernia, they would likely be able to self-diagnose a new defect [10]. Some items in the present questionnaire (Q1, Q2, Q5) were dedicated to detecting recurrences. In the case of a positive answer, the patient was recommended to attend for clinical review. A recent study by Baucom et al. suggested that PROM may be of value in detecting recurrences [33]: The present results do at least provide evidence as to the safety, efficacy, and ease of implantation of SCM, which supports its continued use. Further research is needed to evaluate the long-term outcomes of SCM hernia repair, with randomized studies to assess how it compares to other mesh products.

\section{Conclusion}

Repair of primary and incisional abdominal hernias using Symbotex ${ }^{\mathrm{TM}}$ composite mesh (SCM) is safe, with high patient satisfaction at 2-year follow-up. Complications were limited and non-life-threatening. There was only one questionable hernia recurrence. 12 patients reported some form of usually mild discomfort, pain, or loss of sensitivity at final assessment. These results support the long-term efficacy of SCM.

Acknowledgements Hernie Club Members: M. Antor: CHU Rouen, Rouen, France; M. Beck: Clinique Ambroise Paré, Thionville, France; C. Barrat: Hôpital Universitaire Jean Verdier, Bondy, France (deceased); C. Berney: Bankstown-Lidcombe Hospital, Sydney, Australia; D. Binot: Clinique Côte d'Opale, Boulogne sur Mer, France; D. Blazquez: Clinique Jeanne d'Arc, Paris, France; A. Bonan: Hôpital Privé d'Antony, Antony, France; J. Bousquet: Hôpital privé de la chataigneraie, Montpellier, France; O. Cas: Centre Médico Chirurgical-Fondation, Wallerstein, Arès, France; A. Dabrowski: Clinique de Saint Omer, Saint Omer, France; H. Demian: CHU de la Croix Rousse, Lyon, France ; V. Dubuisson: CHU Pellegrin Bordeaux, Bordeaux, France; A. Champault-Fezais: Groupe Hospitalier Paris. St Joseph, Paris, France; P. Chastan: Bordeaux, France; J.-M. Chollet: Hôpital Privé d'Antony, Antony, France; J.-P. Cossa: CMC Bizet, Paris, France; S. Demaret: Clinique Saint Vincent, Besançon, France; T.Delaunay: Clinique St Hilaire, Rouen, France; F. Drissi: CHU Nantes, Nantes, France; T. G. Dugue: Clinique de Saint Omer, Saint Omer, France; G. Fromont: Clinique de Bois Bernard, Bois Bernard, France; J.-F. Gillion: Hôpital Privé d'Antony, Antony, France; C. Jacquin: CH du Prado, Marseille, France; F. Jurczak: Clinique Mutualiste, Saint Nazaire, France; H. Khalil: CHRU Rouen, Rouen, France; M.V. LaunaySavary: CHU Pellegrin Bordeaux, Bordeaux, France; P. Ledaguenel: Clinique Tivoli, Bordeaux, France; M. Lepère: Clinique Saint Augustin, Nantes, France; D. Lépront: Polyclinique de Navarre, Pau, France; N. Le Toux: Clinique Jeanne d'Arc, Paris, France; J. H. Longeville: Clinique de Nevers, Nevers, France; E. Magne: Clinique Tivoli, Bordeaux, France; P. Ngo: Hôpital Américain, Neuilly, France; O. Oberlin: Croix St Simon Diaconesses, Paris, France; X. Pavis d'Escurac: Strasbourg, France; J. B. Putinier: CHM Grenoble, Grenoble, France; Y. Renard: CHRU Reims, Reims, France; B. Romain: CHU de Strasbourg, Strasbourg, France; S. Roos: Clinique Claude Bernard, Albi, France; M. Soler: Polyclinique Saint Jean, Cagnes-sur- Mer, France; J.-M. Thillois: Hôpital Privé d'Antony, Antony, France; P. Tiry: Clinique de Saint Omer, Saint Omer, France; R. Verhaeghe: MCO Cote d'Opale, Boulogne sur Mer, France; P. Vu: Hôpital Privé Marne-laVallée-Brie-sur- Marne, France; C. Zaranis: Clinique de la Rochelle, La Rochelle, France.

Author contributions All authors qualify for authorship under the ICMJE Recommendations (http://www.icmje.org): JFG: study design, study recruitment, study analysis, manuscript writing, critical discussion, and approval of the final manuscript. ML: Study recruitment, manuscript writing, critical discussion, and approval of the final manuscript. CB, OC, AD, FJ, HK, CZ: Study recruitment, manuscript review, and approval of the final manuscript.

Funding Covidien LP (Trevoux, France) provides clinical study support and data analysis to the independent Club Hernie registry on a pro bono basis. This paper was developed under the authors' guidance by Steven Walker, Robert Davies, Ananya Malladi and Harry O'Connor at Stgilesmedical London \& Berlin (http://www.stgmed.com). Funding of St Giles Medical was provided by Medtronic plc. 


\section{Compliance with ethical standards}

Conflict of interest JG declares conflict of interest not directly related to the submitted work (x). ML declares conflict of interest not directly related to the submitted work (x). CB declares conflict of interest not directly related to the submitted work (x). OC declares conflict of interest not directly related to the submitted work (x). AD declares conflict of interest not directly related to the submitted work (x). FJ declares no conflict of interest. HK declares conflict of interest not directly related to the submitted work (x). CZ declares conflict of interest not directly related to the submitted work (x). J.F. Gillion: President of ClubHernie and organizer of the annual Mesh Congress. He has received expert honorarium and/or non-financial support from Medtronic, Bard Davol, Cousin Biotech, MSD and Gore. M. Lepere: Non-financial support from Medtronic. C. Barrat: Consultant for Medtronic. Grants and/ or fees from Bard Davol, Johnson \& Johnson, MSD and Gore. O. Cas: Research funding from Medtronic. A. Dabrowski: Non-financial support from Medtronic. F. Jurczak: No conflict of interest. H. Khalil: Non-financial support from Medtronic. C. Zaranis: Relations with Bard DAvol, Cousin Biotech, MSD and Gore. RE, outside the submitted work.

Trial registration, ethical approval, and informed consent This study was registered on http://www.clinicaltrials.gov (NCT02206828). The French national ethics committee, the Comité de Protection des Personnes (CPP), was informed, and it subsequently issued a study waiver (CPP Sud Est III Lyon-File QH 15/2014) due to the registry nature of the project.

Human and animal rights All participants received written information about the study, but in view of the study waiver, signed consent was not required. This study did not involve any studies on animals.

Editorial support It was provided by Patrice Becker, Mathilde Lourd, Djamila Bennat and Nicholas Paquette at Medtronic.

Open Access This article is distributed under the terms of the Creative Commons Attribution 4.0 International License (http://creativeco mmons.org/licenses/by/4.0/), which permits unrestricted use, distribution, and reproduction in any medium, provided you give appropriate credit to the original author(s) and the source, provide a link to the Creative Commons license, and indicate if changes were made.

\section{References}

1. Langbach O, Bukholm I, Benth JS, Rokke O (2016) Long-term quality of life and functionality after ventral hernia mesh repair. Surg Endosc 30(11):5023-5033

2. Gillion JF, Sanders D, Miserez M, Muysoms F (2016) The economic burden of incisional ventral hernia repair: a multicentric cost analysis. Hernia 20(6):819-830

3. Kroese LF, Kleinrensink GJ, Lange JF, Gillion JF, Hernia C (2018) External validation of the European Hernia Society Classification for postoperative complications after incisional hernia repair: a cohort study of 2191 patients. J Am Coll Surg 226(3):223-229 e1

4. Awaiz A, Rahman F, Hossain MB, Yunus RM, Khan S, Memon B et al (2015) Meta-analysis and systematic review of laparoscopic versus open mesh repair for elective incisional hernia. Hernia 19(3):449-463
5. Sauerland S, Walgenbach M, Habermalz B, Seiler CM, Miserez M (2011) Laparoscopic versus open surgical techniques for ventral or incisional hernia repair. Cochrane Database Syst Rev 3:Cd007781

6. van Ramshorst GH, Eker HH, Hop WC, Jeekel J, Lange JF (2012) Impact of incisional hernia on health-related quality of life and body image: a prospective cohort study. Am J Surg 204(2):144-150

7. Kaufmann R, Halm JA, Eker HH, Klitsie PJ, Nieuwenhuizen J, van Geldere D et al (2018) Mesh versus suture repair of umbilical hernia in adults: a randomised, double-blind, controlled, multicentre trial. Lancet 391(10123):860-869

8. Heniford BT, Park A, Ramshaw BJ, Voeller G (2003) Laparoscopic repair of ventral hernias: nine years' experience with 850 consecutive hernias. Ann Surg 238(3):391-399 (discussion 9-400)

9. LeBlanc K (2016) Proper mesh overlap is a key determinant in hernia recurrence following laparoscopic ventral and incisional hernia repair. Hernia 20(1):85-99

10. Hauters P, Desmet J, Gherardi D, Dewaele S, Poilvache H, Malvaux $P$ (2017) Assessment of predictive factors for recurrence in laparoscopic ventral hernia repair using a bridging technique. Surg Endosc 31(9):3656-3663

11. Nardi M Jr, Millo P, Brachet Contul R, Lorusso R, Usai A, Grivon $M$ et al (2017) Laparoscopic ventral hernia repair with composite mesh: Analysis of risk factors for recurrence in 185 patients with 5 years follow-up. Int J Surg 40:38-44

12. Balique JG, Benchetrit S, Bouillot JL, Flament JB, Gouillat C, Jarsaillon $P$ et al (2005) Intraperitoneal treatment of incisional and umbilical hernias using an innovative composite mesh: fouryear results of a prospective multicenter clinical trial. Hernia 9(1):68-74

13. Chelala E, Barake H, Estievenart J, Dessily M, Charara F, Alle JL (2016) Long-term outcomes of 1326 laparoscopic incisional and ventral hernia repair with the routine suturing concept: a single institution experience. Hernia 20(1):101-110

14. Lepere MGJ, Barrat C, Cas O, Dabrowski A, Jurczak F et al (2017) First year results on the use of a monofilament polyester mesh with collagen barrier for primary and incisional ventral hernia repair. Int Surg. https://doi.org/10.9738/INTSURG-D-1700113.1

15. Weldring T, Smith SM (2013) Patient-reported outcomes (PROs) and patient-reported outcome measures (PROMs). Health Serv Insights 6:61-68

16. Kroese LF, Gillion JF, Jeekel J, Kleinrensink GJ, Lange JF, Hernia-Club M (2018) Primary and incisional ventral hernias are different in terms of patient characteristics and postoperative complications - a prospective cohort study of 4565 patients. Int $\mathrm{J}$ Surg 51:114-119

17. Palanivelu C, Rangarajan M, Parthasarathi R, Madankumar MV, Senthilkumar K (2008) Laparoscopic repair of suprapubic incisional hernias: suturing and intraperitoneal composite mesh onlay. A retrospective study. Hernia 12(3):251-256

18. Ammaturo C, Bassi UA, Bassi G (2010) Outcomes of the open mesh repair of large incisional hernias using an intraperitoneal composite mesh: our experience with 100 cases. Updates Surg 62(1):55-61

19. Daabiss M (2011) American Society of Anaesthesiologists physical status classification. Indian J Anaesth 55(2):111

20. Muysoms FE, Miserez M, Berrevoet F, Campanelli G, Champault GG, Chelala E et al (2009) Classification of primary and incisional abdominal wall hernias. Hernia 13(4):407-414

21. Gillion JF, Chollet JM (2013) Chronic pain and quality of life (QoL) after transinguinal preperitoneal (TIPP) inguinal hernia repair using a totally extraperitoneal, parietalized, Polysoft (R) 
memory ring patch: a series of 622 hernia repairs in 525 patients. Hernia 17(6):683-692

22. Clavien PA, Barkun J, de Oliveira ML, Vauthey JN, Dindo D, Schulick RD et al (2009) The Clavien-Dindo classification of surgical complications: five-year experience. Ann Surg 250(2):187-196

23. Zhang Y, Zhou H, Chai Y, Cao C, Jin K, Hu Z (2014) Laparoscopic versus open incisional and ventral hernia repair: a systematic review and meta-analysis. World J Surg 38(9):2233-2240

24. Rogmark P, Petersson U, Bringman S, Ezra E, Osterberg J, Montgomery A (2016) Quality of life and surgical outcome 1 year after open and laparoscopic incisional hernia repair: PROLOVE: a randomized controlled trial. Ann Surg 263(2):244-250

25. Liang MK, Clapp M, Li LT, Berger RL, Hicks SC, Awad S (2013) Patient satisfaction, chronic pain, and functional status following laparoscopic ventral hernia repair. World J Surg 37(3):530-537

26. Langbach O, Bukholm I, Benth JS, Rokke O (2015) Long term recurrence, pain and patient satisfaction after ventral hernia mesh repair. World J Gastrointest Surg 7(12):384-393

27. Guerin G, Turquier F (2013) Impact of the defect size, the mesh overlap and the fixation depth on ventral hernia repairs: a combined experimental and numerical approach. Hernia 17(5):647-655

28. Baker JJ, Oberg S, Andresen K, Rosenberg J (2018) Decreased reoperation rate for recurrence after defect closure in laparoscopic ventral hernia repair with a permanent tack fixated mesh: a nationwide cohort study. Hernia 22(4):577-584

29. Groene SA, Prasad T, Lincourt AE, Augenstein VA, Sing R, Heniford BT (2016) Prospective, multi-institutional surgical and quality-of-life outcomes comparison of heavyweight, midweight, and lightweight mesh in open ventral hernia repair. Am J Surg 212(6):1054-1062

30. Elango S, Perumalsamy S, Ramachandran K, Vadodaria K (2017) Mesh materials and hernia repair. Biomedicine (Taipei) 7(3):16

31. Kyle-Leinhase I, Kockerling F, Jorgensen LN, Montgomery A, Gillion JF, Rodriguez JAP et al (2018) Comparison of hernia registries: the CORE project. Hernia 22:561-575

32. Helgstrand F, Rosenberg J, Kehlet H, Strandfelt P, Bisgaard T (2012) Reoperation versus clinical recurrence rate after ventral hernia repair. Ann Surg 256(6):955-958

33. Baucom RB, Ousley J, Feurer ID, Beveridge GB, Pierce RA, Holzman MD et al (2016) Patient reported outcomes after incisional hernia repair-establishing the ventral hernia recurrence inventory. Am J Surg 212(1):81-88

Publisher's Note Springer Nature remains neutral with regard to jurisdictional claims in published maps and institutional affiliations. 